\title{
The evolution of life in the Universe: are we alone?
}

\author{
Jill C. Tarter \\ SETI Institute, 515 N Whisman Road, Mountain View, CA 94043, USA \\ email: tarter@seti.org
}

\begin{abstract}
In his book Plurality of Worlds, Steven J. Dick (1984) has chronicled the millennia of discourse about other inhabited worlds, based upon deeply held religious or philosophical belief systems. The popularity of the idea of extraterrestrial life has waxed and waned and, at its nadir, put proponents at mortal risk. The several generations of scientists now attending this General Assembly of the International Astronomical Union at the beginning of the $21^{\text {st }}$ century have a marvelous opportunity to shed light on this old question of habitable worlds through observation, experimentation, and interpretation, without recourse to belief systems and without risking their lives (though some may experience rather bumpy career paths). The newly-named and funded, multi-disciplinary field of astrobiology is extremely broad in its scope and is encouraging IAU members to learn and speak the languages of previously disparate disciplines in an attempt to answer the big picture questions: 'Where did we come from?', 'Where are we going?', and 'Are we alone?' These are questions that the general public understand and support, and these are questions that are attracting students of all ages to science and engineering programs. These questions also push the limits of modern instrumentation to explore the cosmos remotely across space and time, as well as to examine samples of interplanetary space returned to the laboratory and samples of distant time teased out of our own Earth.

Within my personal event horizon, the other planetary systems long-predicted by theorists have been uncovered, along with many whose structures were not predicted. The 'just-so' conditions requisite for the comfort of astronomers have been understood to be only a very narrow subset of the conditions that nurture extremophilic, microbial life. Thus the potentially habitable real estate beyond Earth has been greatly expanded and within the next few decades it may be possible to detect the biosignatures or technosignatures of inhabitants on distant worlds, should there be any.
\end{abstract}

\section{Introduction}

Since we are here, it must be the case that this Universe, at least, is biofriendly. I leave the question of whether or not there are other universes to those of you in the audience far more qualified than I am. I will content myself with wondering just how biofriendly this Universe is, and whether it is possible to find scientific evidence of the existence of life elsewhere.

Humans have been asking the 'Where did we come from?' question throughout oral and recorded histories. Every culture has its own creation myth, sometimes more than one. The scientific culture is no exception; we have our own scientific creation myth called cosmology. Because our myth is bolstered by exquisite observational evidence and is capable of predictive precision, we correctly view it in a different light than other creation myths; though some fundamental cosmological questions remain unanswered, and we do not know whether they ever can be (Greene 2000). 
The first widely published science fiction work (Shelley 1818) in the early $19^{\text {th }}$ century is a good marker for human interest in the 'Where are we going?' question. In this audience, we study the fate of our Universe as a result of its dark-energy-driven, accelerated expansion. Modern cultures, experiencing accelerated change in their lives due to technological innovation, have begun speculating about, and manipulating, the future course of the human race. Today none of us can say whether there will yet be many long and happy chapters to the story of humanity, or whether this history will terminate abruptly, or perhaps purposefully evolve into something other than human. But contrary to our ancestors, we expect that the future will be different and we struggle to anticipate the change.

Since my graduate student days, two phenomena have been given more and more attention: extremophiles and extrasolar planets. Both are relevant to the question of other habitable worlds and inhabitants.

\section{Astrobiology}

Astrobiology is the science that deals with the origin, evolution, distribution, and future of life in the Universe. It has been successful in bringing together scientific specialists from many different disciplines to tackle these big-picture questions. Many of my colleagues at the SETI Institute are astrobiologists, studying organisms living in extreme conditions (by human standards) in an attempt to better understand the origin of life on Earth and the potential habitable real estate for life beyond Earth. In the past few decades we have expanded the range of conditions recognized as suitable for life. Life is no longer confined between the boiling and freezing points of water. Hyperthermophiles live at high temperatures (and sometimes also high pressures), the current record holder being archean microbial Strain 121 that thrives at $121^{\circ} \mathrm{C}$ metabolizing iron, but it can survive up to $130^{\circ} \mathrm{C}$ (Kashefi \& Lovley 2003). At the other extreme, the psychrophilic bacterium Psychromonas ingrahamii survives and reproduces (very slowly) at $-12^{\circ} \mathrm{C}$ in the ice off Point Barrow, Alaska (Breezee et al. 2004). Macroscopic ice worms occupy and move through the Alaskan glaciers as well as the methane ice seeps on the floor of the Gulf of Mexico using natural antifreeze to protect their cellular structures (Fisher et al. 2000). Sunlight, once argued to be the source of energy for all life, is completely absent miles beneath the surface of the ocean, around the deep hydrothermal vents, where a rich and diverse community of organisms thrives in the dark, at enormous pressures. Small blind shrimp there have developed IR-sensing eye spots to navigate the vent environs or to travel from one vent to another using their thermal signatures (Pelli \& Chamerlain 1989). Some chemical process (or processes) within the vents also produces minute quantities of visible light that are harvested by green sulfur bacteria for photosynthesis even though many hours go by between photons (Beatty et al. 2005). Humans increasingly protect themselves from exposure to UV radiation as the protective layer of atmospheric ozone thins, since our DNA lacks sufficient repair mechanisms to survive intense radiation environments. Yet organisms inhabiting the highest freshwater lakes on Earth, in the caldera of the Lincancabur volcano overlooking the Atacama desert, have adapted to the huge UV load their altitude and evaporating environment present (Cabrol et al. 2005). Colleagues from the SETI Institute free-dive in these lakes each austral spring to catalog these organisms and study the DNA repair mechanisms they have elaborated. If life once occupied Mars, similar mechanisms might have been employed by organisms seeking to survive the dual stresses of increased radiation and desiccation due to loss of planetary atmosphere. Even more spectacular, Deinococcus radiodurans can withstand millions of rads of hard radiation because its DNA repair mechanisms 
are so effective. This skill probably evolved, not because it encountered naturally high radiation environments, but because desiccation causes the same sorts of breaks in DNA linkage; a sufficiently robust repair mechanism can endow survival independent of the damage source (Mattimore \& Battista 1996). As a result, this microbe is the focus of many bioremediation programs to deal with radioactive materials, and is being sought in the Atacama to demarcate areas just too dry for life. Neutral $p H$ was once thought essential for life, but acidophiles are plentiful; cyanobacteria and fish can survive at $p H \simeq 4$, but the red alga Cyanidium caldarium and the green alga Dunaliella acidophila can live at $p H<1$ (Rothschild \& Mancinelli 2001). In the ground waters of industrial slag heaps, extremely alkaline-tolerant microbes have been found thriving at a $p H$ of 12.8 (Roadcap et al. 2006). While salt has been used historically to preserve food from decay due to bacterial action, halophilic archean microbes have been found living within pure $\mathrm{NaCl}$ crystals (Rothschild et al. 1994). Astronomers may find it extremely unpleasant to live beyond the 'just right' bounds of our current terrestrial environment, but clearly life has a greater tolerance and no lack of innovative ways of making a living. Within the past few years, we have begun to accept the concept of the 'deep hot biosphere', and to acknowledge that perhaps ten times as much biomass is resident in the crust beneath our feet, as compared to the surface biomass with which we have long been familiar (Gold 1998). All these biological adaptations must inform our searches for life elsewhere in the Universe.

Although it appears that life populated the Earth in a geologic eye blink, following the appearance of liquid water on its surface, and although we have astronomical evidence of ubiquitous organic chemistry together with observations of uniformity in the laws of physics and chemistry through vast stretches of space and time, and although there is much reason to sympathize with Christian De Duve who sees life as a cosmic imperative (De Duve 1996), we cannot yet conclude that life of any form actually exists elsewhere in our Universe. Astrobiologists are engaged in a large number of different efforts to look for the proof of existence.

While no fundamental definition of 'life' exists, nor for that matter of 'planet' (until this particular IAU General Assembly) it is still a valid claim that life-as-we-know-it is a planetary phenomenon. That is, all examples of life show a remarkable genetic connection, suggesting that life originated and evolved on this planet. As it did so, it was profoundly affected by the planetary environment and life, in turn, profoundly affected the planet Earth. Thus a search for life elsewhere necessarily starts with a search of the other planets in our solar system, and a search for exoplanets beyond. Mindful of the adaptability of life to extreme environments, within our own solar system (where we may have some hope of systematic in-situ sampling) we should expand our inspection to any bodies capable of providing raw materials and energy sources that might be exploited by biology of any sort. Thus, in addition to the terrestrial planets (which were all biologically connected during an earlier epoch of planet building and bombardment), we should consider the large icy satellites of Jupiter and Saturn, namely Europa, Ganymede, Callisto, and Enceladus where liquid, briny, water oceans are thought to exist beneath their icy outer crusts. Titan might be a world hosting biology without liquid water as a solvent, though the slow reaction rates there challenge the notions of reproducibility and heritable evolutionary change that often appear in attempts at the definition of life. Perhaps results from the ESA's Venus Express mission will shed light on whether the chemical disequilibria previously noted in the Venusean atmosphere require explanations involving Grinspoon's revival of the Sagan and Salpeter 'sinkers, floaters, hunters and scavengers' (Schulze-Makuch et al. 2004). For the foreseeable future NASA will continue a 'follow the water' strategy, returning to Mars with robots and humans to look for signs of extinct 
life from a wetter, warmer epoch or even for subsurface extant life. In addition they will try to understand whether a biotic mechanism must be invoked to explain the claimed, current source of an inhomogeneous distribution of atmospheric methane (Mumma et al. 2005). Subsequent missions may venture to Europa to verify the existence of a massive water ocean and to examine the tantalizing discolorations near the surface cracks that could be the end products of organic molecule irradiation (Dalton et al. 2003), and then, later still, return to make a sterile penetration of the ice and search for life in the water below. No missions are yet planned for the other bodies of interest, but from the point of view of astrobiology, all such missions would be warranted.

\section{Exoplanets}

Moving out beyond the solar system, the focus will be on exoplanets. The current prize being the detection of, and susequent imaging of, a terrestrial-mass planet within the so-called 'habitable zone' of its host star. While traditionally referring to the region of space where a planet might be expected to have surficial liquid water, the habitable zone should now be enlarged to include giant planets with potentially habitable moons, and other environments that might host extremophiles.

Today, exoplanets are primarily detected by indirect techniques: astrometry, radial velocity studies, transits, and gravitational micro-lensing. Most of the planets discovered are giants; a $5.5 \mathrm{M}_{\text {Earth }}$ planet from a micro-lensing event is the lowest mass to date (Beaulieu et al. 2005). 'Hot Jupiters' in short-period orbits and an abundance of higheccentricity orbits were surprising discoveries, though we are beginning to have reasonable explanations based on interactions with viscous protoplanetary disks and a version of cosmic billiards. Initially it was assumed that the presence of hot Jupiters would doom any terrestrial planets within the habitable zone, but recent studies by Raymond et al. (2006) argue that it is possible to have wet, terrestrial planets, even though a Jupitermass planet has migrated through their orbital radii on its way towards the star. We believe that the absence to date of a true Jupiter analog (in a circular orbit at $5 \mathrm{AU}$ distance from a solar-type star) is the result of an observational bias that will be overcome once sufficiently long datasets from high-precision, radial-velocity instruments have been collected. Radial-velocity instruments with precisions of $1 \mathrm{~m} / \mathrm{s}$ are currently in operation at Lick, Keck, and La Silla Observatories, and the first purpose-built observatory, the Automated Planet Finder is, nearing completion. Precise photometric measurements of transit events may soon yield terrestrial mass planets from the CoRot spacecraft to be launched later this year, or the more capable Kepler mission to launch in 2008. Of note: Kepler is sized such that a failure to find any Earth-sized planets will be a significant null result, drastically changing our ideas about the processes that form planetary systems. The Space Interferometry Mission (SIM) will achieve the astrometric precision necessary to detect planets of a few Earth-masses orbiting the closest stars. Recent progress has been made in networking together a significant number of smaller, optical observatories. An alert system makes possible rapid follow-up to detect the brief microlensing events caused by a propitiously placed planet orbiting a foreground star during the gravitational lensing of a background star. This should greatly enhance the detection rate of exoplanets, though further observations of these particular planetary bodies will not be possible. The near-term future holds promise for the detection of other Earths, large moons of gas-giant planets, and other potentially habitable cosmic real estate. The next obvious question is; will they be inhabited? 


\section{Exolife}

Just how exactly like the Earth does another environment have to be in order to host life? Ward \& Brownlee (2001) have argued that an exact duplicate of the terrestrial environment, its history, large moon, and giant-planet shields are required for anything beyond microbial life. However, Darling (2001) reviews the arguments and concludes that other astronomers might exist on many worlds. In fact the evidence is consistent with life, including intelligent life, existing on many worlds or exclusively only on Earth; there is as yet no evidence. We have an example of 'one', we cannot know from tracing the detailed history of that single example what the branching ratio might be for the experiment of life; how many other ways might things have gone but didn't, at what rate, with what end result? The number "two" will be all important in answering this question - as in the second example of an independent origin of life. In 1996 McKay et al. (1996) claimed that the best explanation for features found in the Allen Hills 84001 meteorite (actually an ancient piece of Mars) was that the features represented a fossilized form of life. This claim stirred up great controversy and subsequently, very innovative experimentation. Additionally it reminded us that the detection of life elsewhere in the solar system must necessarily raise the further questions of biological infection vs. second genesis. While both answers are intriguing, only the latter has significant implications for life beyond the solar system.

Within the solar system, in-situ measurements and sample return missions perhaps have the opportunity of detecting life by knowing-it-when-we-see-it. Observations searching for life elsewhere must rely on predetermined biosignatures or technosignatures; if the wrong filter is assumed and applied, it may miss valid evidence that is there. Similarly, in non-terrestrial environments, unappreciated, abiotic causes may be explanation for the results of any observations, thus leading to the potential for false positives.

It seems impossible to avoid this particular trap of being $21^{\text {st }}$ century humans. In seeking life, or its technological by-products, we cannot search for what we cannot conceive, and it is also impossible to guarantee that we will correctly interpret what we find. This conundrum has been shared by all past explorers. Those who were successful pushed ahead, with the tools at their disposal, or tools they invented. As my colleague Seth Shostak is fond of saying "Columbus didn't wait for a 747 to cross the Atlantic". Neither should astrobiologists.

\section{Biosignatures}

The Viking exobiology experiments on Mars in 1976 were the first life-detection attempts on another world. While most scientists agree that Viking detected evidence of an unexpected superoxide soil chemistry, a few still believe that the results are consistent with a biological source (Levin 1997). The Viking laboratory-in-a-box experimental suite attempted to find life by looking for it with cameras (nobody looked back), looking for a changes in gas composition after soil samples were moistened and then 'fed' nutrients, providing soil samples with labeled nutrients and water then looking for metabolism in the form of $\mathrm{C}^{14} \mathrm{O}_{2}$ release (this is the contested result), and using pyrolized soil samples as input into a gas chromatograph (no mass fragments corresponding to organic compounds were detected). Whether or not the Viking results were indeed due to unexpected soil chemistry, it is clear from our improved understanding of extremophiles, that Viking would have failed to detect many different me tabolic pathways based on, e.g., Fe, S, or H that were not considered three decades ago. The current strategy for the Martian rovers (Sojourner, then Spirit and Opportunity) is one of 'following the water'. Having verified 
that early Mars did have flowing surface water, the current topic of debate is how much, for how long, and where is it now (Squyres et al. 2006)? The Martian Science Laboratory $\dagger$ will be the next on the scene, trying to detect extant biology and the by-products of biological activities on the surface and as far below the surface as the next generation of drilling technologies can achieve. Experiments capable of detecting metabolic strategies of life-as-we-know-it are being tested in the remotest and least habitable areas on Earth, but Viking reminds us that there is no guarantee these particular signatures will be all-inclusive or indicative of any Martian life-as-we-don't-yet-know-it.

Moving out beyond the Solar System, the next step will be to attempt to conduct a chemical assay of the atmosphere of any terrestrial planet imaged in orbit around nearby stars. Transiting hot Jupiters have already permitted the first analysis of chemical constituents of exoplanet atmospheres. Observations of HD 209458b reveal sodium, hydrogen, oxygen and carbon in an extended atmosphere and/or escaping from the planet (Vidal-Madjar et al. 2004). The Terrestrial Planet Finder (TPF) being studied by NASA and the Darwin constellation under development by ESA will eventually launch during the first quarter of the $21^{\text {st }}$ century, perhaps combined as an international mission. Telescopes on these spacecraft are intended to suppress the light from a central star, using either an occulting coronagraph at visible wavelengths or interferometric nulling in the IR, thereby achieving a point spread function capable of spatially resolving and directly detecting reflected starlight from any terrestrial exoplanets orbiting within the stellar habitable zone: contrast ratios as great as $10^{-10}$ are required $\neq$. Once an image has been formed, additional observations will attempt to collect sufficient photons to reveal absorption lines in the exoplanet spectrum due to trace atmospheric constituents that might be clues to the presence of biology on the planetary surface.

As difficult a technical challenge as implementing these spacecraft will be, perhaps an even greater challenge will be deciding what spectral signature(s) does or does not constitute a reliable biomarker. Using the present Earth as an example, chemical disequilibrium is one very promising sign. The coexistence of molecular oxygen and methane (more than 30 orders of magnitude out of equilibrium) in our current atmosphere is the direct result of photosynthetic cyanobacteria and plants as well as the fermenting bacteria within termites, ruminants, and rice paddies (Lovelock 1965). But the paleoearth would have presented a very different picture during the billions of years when life was present, but had not yet participated in the elevation of atmospheric $\mathrm{O}_{2}$ levels; for that world, we must ask about the undeniable biosignatures of methanogens? Additionally, one must ask if these biosignatures are not only necessary, but sufficient - are there no abiotic processes that can yield the same result? For exoplanets, the harsh realities of the remote observational circumstances are further challenges. The available spectral coverage on any single mission will be limited; it does not now seem feasible to simultaneously observe the visible bands of $\mathrm{O}_{2}$ and the thermal IR signature of $\mathrm{CH}_{4}$ in one instrument. All observations of atmospheric chemistry will be photon limited, and therefore only the broadest and deepest absorption features will be usable. Detectable amounts of $\mathrm{O}_{3}$ (at $\lambda 9.3 \mu \mathrm{m})$ in the atmosphere of an exoplanet believed to have liquid water on its surface (indicative of an active carbonate-silicate cycle that would quickly drawdown any initial reservoir of ozone) is currently the favored biosignature for complex life-as-weknow-it when the primary is a Sun-like star (Leger et al. 1999). When the primary is an M-type dwarf, other molecules such as $\mathrm{N}_{2} \mathrm{O}$, and $\mathrm{CH}_{3} \mathrm{Cl}$ may be detectable along

$\dagger$ http://mars.jpl.nasa.gov/msl/overview/

$\ddagger$ http://planetquest.jpl.nasa.gov/TPF/tpf_index.cfm

I http://www.darwin.rl.ac.uk/overview.htm 
with ozone (Segura et al. 2005). While there are abiotic sources of oxygen, and therefore ozone, an ongoing biological source appears to be required for substantial atmospheric ozone concentrations on a geologically active planet. Any future announcements of atmospheric ozone detection from an exoplanet and a potential linkage to biota are likely to be accompanied by a long list of caveats.

Photon collection from the atmosphere of a terrestrial exoplanet will take a very long time and spatial resolution will be limited to one, or a few, pixels. Therefore, the spectra will be averaged over the full exoplanet disk, and time-averaged by planetary rotation and orbital phase. Clouds, if present, will further dilute any evidence of vegetation signatures such as the 'red edge' of chlorophyll (a sharp increase in leaf reflectance around $700 \mathrm{~nm}$ ) and the differences in albedo or specular reflection caused by land masses and water oceans (Montanes-Rodriguez et al. 2005). To prepare for these future missions, to optimize the onboard spectroscopic instrumentation, and to plan for interpreting realistic data returns, the globally averaged signature of the Earth is being measured in earthshine from the moon (Woolf et al. 2002). In addition, the surface biota and atmosphere of the Earth through time are being used as input to numerical models of exoplanets in orbit around stars of different spectral type (Tinetti et al. 2006). Investigations into the metabolic strategies of microbial extremophiles can also help prepare for the interpretation of possible future spectral biosignatures from life-as-we-don't-yet-know-it. However, spectral absorption features alone are unlikely to distinguish between a future detection of alien microbes or mathematicians.

\section{Technosignatures}

'Are we alone?' is really a loaded question. When humans pose it, they are generally not asking whether another world is teeming with bacterial analogs. The detection of life on another world (extant or extinct) is a real possibility within the lifetimes of many of us at this General Assembly. That is a thrilling possibility. Detection of irrefutable biosignatures will provide the pivotal 'number two' in the record of life in the Universe, but at a very deep level, humans want to know whether other intelligent creatures also view the cosmos and wonder how they came to be. Like the term 'life', there is really no acceptable definition of 'intelligence'. Nevertheless we may be able to remotely deduce its existence over interstellar distances. If we can find technosignatures - evidence of some technology that modifies its environment in ways that are detectable - then we will be permitted to infer the existence, at least at some time, of intelligent technologists. As with biosignatures, it is not possible to enumerate all the potential technosignatures of technology-as-we-don't-yet-know-it, but we can define systematic search strategies for equivalents of some $21^{\text {st }}$ century terrestrial technologies. Recognizing that we are a very young technology in a ten billion year old galaxy, we should continue to explore our Universe utilizing every wavelength and every information-bearing particle, with all of the observational tools we can construct, remaining vigilant for anomalies that might be new 'Class A' astrophysical phenomena (Harwit 1981) or might just be the detectable consequences of someone else's astro-engineering project or catastrophe. That is, we should pursue astronomy with vigor and in the spirit of Jocelyn Bell Burnell (1977), letting no bit of 'scruff' go unheeded.

SETI (the Search for ExtraTerrestrial Intelligence) is that subspecialty of astrobiology that currently conducts systematic explorations for other technology-as-we-know-it; primarily searches for electromagnetic radiation. SETI predates the current field of astrobiology, beginning life as a valid field of exploratory science in 1959 with the publication of the first paper on this subject in a refereed journal (Cocconi \& Morrison 1959). This 
first paper advocated a search for radio signals, but the suggestion of searching for pulsed optical laser signals followed shortly thereafter in 1961 (Townes \& Schwartz 1961). SETI provides another plausible avenue for discovering habitable worlds by attempting to detect the actions of technological inhabitants. More than a hundred SETI searches can be found in the literature (Tarter 2001) beginning with Project Ozma in 1960 (Drake 1960), the first search for radio signals from nearby Sun-like stars. While this may seem like a large effort, the sum total of all these investigations has covered only a minute fraction of the search-parameter space.

Information can be encoded with photons for the benefit of the transmitting technology or to deliberately attract the attention of another civilization. While it may be possible for us to detect unintentional leakage radiation from another technology, deliberate signals, transmitted to be detectable, are the most likely to be detected. Furthermore, any detectable signals will have originated from a technology far older than our own. There may be no other detectable technological civilizations in the Milky Way or beyond, but if there are others, we can be confident that we are youngest. On Earth, we've had at least one viable technology for interstellar communication for about a hundred years, prior to that our technology was undetectable at a distance. Today we are in a period of rapid exponential improvements in information processing and communication technologies. Whether that exponential will continue indefinitely into a future dominated by machine rather than biological intelligence (the 'singularity' of Kurzweil and other futurists (Kurzweil 2005), or whether the exponential will saturate and sustain due to some resource limitation is currently unknown. There is of course another alternative; that our technology will cease, due to its destruction or deliberate disuse. If technology tends to be a long-lived phenomenon among Galactic civilizations, then statistics favor the detection of signals from a technology during its old age. If technology, in general, is a short-lived phenomenon, then it will be undetectable because the chance that two technological civilizations would be not only co-located, but co-temporal during the 10 billion year history of the Milky Way is vanishingly small. For this reason, Philip Morrison has called SETI the archeology of the future. The finite speed of light guarantees (for current understanding of physics and available technologies) that any detected signal will tell us about the transmitter's past, but the detection of any signal tells us that it is possible for us to have a technological future (Mallove 1990). The potential for deliberate disuse of any given technology is another argument against the likelihood of detecting leakage radiation; any transmission mechanism for internal use is likely to be superseded by a more efficient technology within a short time. However, with respect to deliberately transmitted signals, a long-term strategy is implicit (the signal must be there whenever the intended receiver evolves to listen, if transmission is to be successful). Furthermore, an older technological civilization might choose a transmission technology that favors detection by emergent technologies (such as ourselves) rather than reflecting its own capabilities. Or an older technological civilization might choose to do the opposite, that is to adopt a transmission strategy that is only detectable by another technological civilization with some minimum level of competence, not by us. Ab initio, we cannot unravel alien motivation, we can only choose to search, or not to search, using the technologies within our grasp. Gott (1993) has used a statistical argument based on the Copernican Principle to suggest that because we find ourselves to be a very young technology in a very old galaxy, it is unlikely that we will become an old technology. Yet if, to the contrary, a long technological existence is indeed in our future, it would be impossible to avoid passing through our current emergent state, at which point we are capable of making Gott's calculation. Considerations of the evolutionary history of galactic supernovae activity, star formation, and metallicity have led to the idea of the galactic habitable 
zone, in which $75 \%$ of the stars are older than the Sun (Lineweaver et al. 2004). Thus, technological civilizations older than our own are indeed a possibility, and we should plan any search strategies with our asymmetric position (we are the youngest detectable technology) in mind.

\section{Exploring the cosmic haystack}

As previously noted, most SETI projects rely on photon detection. Even restricting ourselves to electromagnetic radiation, the cosmic haystack we wish to explore for a signal (by analogy the difficult-to-find needle) is at least nine-dimensional: three dimensions of space and one dimension of time, two senses of orthogonal polarization, one unknown photon frequency, some form of modulation scheme for information encoding, and finally an undetermined minimum sensitivity (because the transmitter power and distance are unknown). Recently it has been suggested that a photon's quantized orbital angular momentum could encode information so that should become another dimension of the cosmic haystack (Harwit 2003). Since there is no near-term, practical implementation of that search strategy for SETI, it will for the moment be counted as part of the dimension dealing with modulation schemes. Contrary to popular belief, since Project Ozma fortysix years ago, we have only managed to pull a few straws from the cosmic haystack for close examination. Most of the time, most SETI searches are off the air. Given the nine-dimensional haystack, searches to date have been unable to set meaningful limits on the existence (or non-existence) of other technological civilizations. Explanations for 'the great silence' are numerous, but in fact they are not yet required. Today the situation is changing as new instruments intended for dedicated SETI use are commissioned and beginning to look at the sky. This is an exciting time because our tools may finally be getting to be commensurate with the magnitude of our task.

As above, we should concentrate on detecting deliberately broadcast signals and try to devise systematic search strategies of sufficient scope that null results will be significant, if positive results continue to elude us. Deliberate signals might be modulated in one of two ways: they could appear to be 'almost astrophysical', or they could appear to be 'obviously technological'. The distinct benefit of the former scheme is that such signals are very likely to be captured as an emergent technology begins to deploy multiple sensors to study the Universe around it. Detectors will be optimized to record signals with just these sorts of characteristics, and they will be entered into databases as pulsars, GRBs, SNs, eclipsing binaries, etc., etc. Detailed study of the individual entries in any such databases will eventually discover that a particular 'pulsar' had a spindown rate of exactly zero (not just momentarily zero, or zero to instrumental accuracy) or that 'pulsar' might glitch back and forth between two precise rotation periods, or the GRBs might end up being aligned in 4-dimensional space time because they were the acceleration events of some matter-anti-matter annihilation rocket traveling through space, or the light curve of an 'eclipsing' binary star turned out to be caused by an occulter(s) with non-spherical cross-section, or widely separated SNs might have synchronized light curves, or .... As an emergent technology, our rapidly improving astronomical observing capability and our curiosity about the cosmos should insure that we eventually discover any such 'almost astrophysical' signals. Almost by definition, the detection of 'obviously technological' signals will require construction of specific instrumentation not available from astronomical observing programs, because the characteristics of the signals are precisely those that we expect nature to be unable to produce. Within this class of signals, SETI has historically concentrated on those signals whose time-bandwidth product approaches the minimum value permitted by the uncertainty principle. At radio frequencies, signals exhibiting 
extreme frequency compression (CW or narrowband pulses) with bandwidths less than the $\sim 300 \mathrm{~Hz}$ exhibited by line-narrowed, satura ted OH masers (Cohen et al. 1987) are being sought. Because the natural background from Galactic synchrotron emission rises rapidly below $1 \mathrm{GHz}$, while the noise from atmospheric water vapor and oxygen contributes above $10 \mathrm{GHz}$, radio SETI searches have a goal of systematically exploring the naturally quiet Terrestrial Microwave Window from $1-10 \mathrm{GHz}$. While there is no natural background at these frequencies except the $3 \mathrm{~K} \mathrm{CMB}$, radio searches must expend significant computing resources to mitigate the effects of human-caused RFI, and giving up pieces of some spectral bands altogether, even at the quietest sites. At optical frequencies, signals exhibiting extreme time compression (broadband laser pulses) are searched for with photon counters having nanosecond rise times, a regime with no known sources of astrophysical background (Howard \& Horowitz 2001). Because interstellar dust begins to absorb optical pulses over distances beyond $\sim 1000 \mathrm{ly}$, it is desirable to extend the optical SETI search into the infrared so that more of the Galaxy becomes accessible. This will happen when, and if, the requisite fast photon counters become available and affordable in the IR. Other modulation schemes employed by current terrestrial communications technologies can produce signals whose statistical properties differ from the Gaussian noise of astrophysical emitters. Detection of this class of signals is far more compute-intensive than detection of the simple $B \tau \simeq 1$ signals. As Moore's Law delivers more affordable computing, efforts are beginning to accommodate searches for more complex signals at radio frequencies.

At any frequency, there are two basic search strategies that can be implemented: move quickly across the sky (or a portion thereof) to cover as much of the spatial dimension of the cosmic haystack as possible, or select individual directions deemed to have a higher a priori probability of harboring a technological civilization and make targeted observations in those directions for longer periods of time. The former strategy minimizes the assumptions about the source of the signal, and is better suited to the case where the location of the transmitter might no longer be associated with the point of origin of the technological civilization. But, in general, the surveys will achieve poorer sensitivity as the result the smaller telescopes and shorter dwell times typical of this strategy, and they are more likely to be out of phase with transient or periodic emitters. By developing a list of plausible targets, it is possible to achieve significantly better sensitivity through integration and signal processing gain for a wider range of signal types. However, if the target list is constructed under the wrong assumptions, detection probabilities are lowered rather than improved. In both cases it is desirable, but seldom affordable, to accomplish the task of signal detection and recognition in real-time, or near-real-time, so that immediate follow up of candidate signals is enabled before they can vary with time, and opportunities for distinguishing between terrestrial and extraterrestrial technologies can be exploited. Discrimination against terrestrial signals can also be improved by the simultaneous use of multiple telescopes at widely separated sites; lack of coincidence or, in the case of narrowband signals, differential Doppler signatures are powerful tools. Since Project Ozma, plausible arguments about 'magic' frequencies, places, and times have been used to constrain the scope of individual searches. After more than four decades there is still divergence among humans, rather than convergence, with regard to the proper magical parameters. With the exception of a timing scheme based on novae and supernovae (Lemarchand 1994), there is little opportunity to synchronize our magical thinking with the extraterrestrial transmitter.

In 2004, the SETI Institute completed Project Phoenix (Backus (2006)), and this summer UC Berkeley's SERENDIP IV system at Arecibo concluded its data taking (Cobb et al. 2000). Phoenix was the privately-funded continuation of a targeted search of 800 nearby 
solar-type stars from 1.2 to $3 \mathrm{GHz}$ begun as the NASA High Resolution Microwave Survey in 1992, and continued by the SETI Institute following congressional termination of NASA SETI funding in 1993. SERENDIP IV was a commensal, random sky survey conducted with a feed and receiver system attached to the end of the Arecibo carriage house arm not being used by the primary observer. A few percent of the data SERENDIP IV collected was subsequently analysed on the pioneering SETI@home distributed computing platform. Until searches begin with the Allen Telescope Array (ATA, see below) in 2007, there remain only a few organized radio SETI observing programs on the air; the new SERENDIP V recorders attached to the ALFA multibeam feed at Arecibot, Project Argus an amateur effort coordinated by the SETI Leaguef, and a SETI Italia project $\uparrow$. None of the current projects are real-time searches, none can follow-up immediately on candidate signals. Over a 5-year period, Harvard's optical SETI targeted search ran on the Wyeth Telescope at the Oak Ridge Observatory completing observations of more than 6000 stars, many of them synchronously with the Fitz-Randolph Observatory at Princeton University as a successful method to suppress the false-positive event rate (Howard et al. 2004). Both sytems are now shut down, but the Harvard group is commissioning a new OSETI observatory for a survey of the sky visible from Oak Ridge (see below). Targeted OSETI systems are continuing to observe at UCSC Lick Observatory (remotely operated from the SETI Institute)\|, at UCB Leuschner Observatory $\dagger$, , and at the Campbelltown Rotary Observatory as part of an astrobiology instructional program at the University of Western Sydneył‡. At this precise moment, observational progress is slow, as new dedicated SETI observing facilities are brought on line.

\section{Observatories for tomorrow}

The SETI Institute and the University of California Berkeley Radio Astronomy Lab have partnered to build the Allen Telescope Array (ATA) in Northern California at the Hat Creek Radio Observatory for the purpose of simultaneously surveying the radio sky for signals of astrophysical and technological origin (DeBoer et al. 2004). Ultimately the array will consist of 350 antennas, each $6.1 \mathrm{~m}$ in diameter, extending over a maximum baseline of $900 \mathrm{~m}$. The ATA will do for the radio sky what the Sloan Digital Sky Survey has recently done for the optical sky. And it will do it so rapidly that it will also provide the first systematic look at the transient radio universe. The ATA provides simultaneous access to any frequency between $500 \mathrm{MHz}$ and $11.2 \mathrm{GHz}$, a system temperature $\sim 50 \mathrm{~K}$, four separately tunable intermediate frequency channels feeding a suite of signal processing backends. The backend instrumentation can produce wide-angle radio images of the sky with $\sim 20,000$ resolution pixels and 1024 spectral channels per pixel, and at the same time, study up to 32 point sources of interest within its large field of view using phased up beams at up to four different frequencies. With 61,075 baselines feeding two spectral-imaging correlators, the ATA is a superb radio snapshot camera, yielding maps with exceptional image fidelity. This new approach to commensally sharing the sky allows SETI and traditional radio astronomical science to both utilize the telescope nearly full time. For efficient sky 'sharing' a large list of SETI target stars is essential. Two initial catalogs of stars capable of hosting habitable planets, or 'hab stars', have been created

\footnotetext{
$\dagger$ seti.berkeley.edu/casper/papers/2004-07-10_Iceland_SV_poster.pdf

$\ddagger$ http://www.setileague.org/

ब http://www.seti-italia.cnr.it/

\|http://seti.ucolick.org/optical/

$\dagger \dagger$ http://seti.ssl.berkeley.edu/opticalseti/

$\ddagger$ http://www.atnf.csiro.au/pasa/17_2/bhathal/
} 
from data provided in the Hipparcos and Tycho catalogs (Turnbull \& Tarter 2003). The $\sim 250,000$ stars in the current habstar catalogs will provide a few stellar targets in every array field of view at lower frequencies. Future results from the Gaia mission will permit expansion of these catalogs by a factor of 4 or more in order to maintain observing efficiency at the higher frequencies with smaller primary fields of view. The current generation of SETI signal processing backends (Prelude) are based on PCs, each with a special accelerator card, and provide over 200 millions channels of $1 \mathrm{~Hz}$ resolution channels that can be spread over as many as three dual-polarization phased array beams. The output data from every ATA beam is delivered in a standard IP format. Development is now ongoing to convert to the next generation of SETI signal processing equipment (SETI on the ATA or SonATA) as 'software only' implementations within generic computing clusters. Since the full $11 \mathrm{GHz}$ bandwidth analog signal is transported from the antennas to a central processing lab next to the array, future improvements in the cost performance ratios of digitizers and other Moore's Law devices promise improved throughputs and observing speeds. Over the next observational decade, it should be possible to observe about a million SETI target stars through the terrestrial microwave window from 1 to $10 \mathrm{GHz}$. Today there are 42 antennas in the array, and they are spaced fairly close together for ease of commissioning. Therefore, the first SETI search that will be conducted with the ATA-42 will be a survey of 20 square degrees along the Galactic plane in the vicinity of the center and will cover the 1420 to $1720 \mathrm{MHz}$ 'waterhole'. Along these lines of sight, the survey will interrogate $\sim 10^{10}$ stars, most of them distant, searching for strong technological signals. At the distance of the Galactic Center this survey would detect a transmitter with a power equivalent to 20,000 Arecibo planetary radars. A targeted search of a million nearby stars with the full 350 element array should have the sensitivity to detect transmitters with powers ranging from that of current terrestrial ionospheric backscatter radars up to the Arecibo planetary radar, depending on the stellar distance. The first radio science to be undertaken with the ATA- 42 will be a $5 \mathrm{GHz}$ survey of $10^{4}$ square degrees of the sky (overlapping with the SDSS) using the spectral imaging correlator to search for transients. Selected sky patches of 100, and 10 square degrees, will be revisited weekly and daily. In this way, it should be possible to characterize the temporal variability of the radio sky on a range of timescales and provide finding lists of variable sources that will inform a number of different scientific studies in the community. The standard IP protocol data output for the phased array beams invites external users to construct their own backend processors for use on the ATA to enlarge the amount of commensal observing accomplished with the array.

The ATA is the first attempt to manufacture a radio telescope by taking advantage of cost discounts from economies of scale, consumer off-the-shelf components (primarily from the telcom industry), and inexpensive commercial manufacturing technologies. The least expensive way to build such an array is all at once. Paul Allen, Nathan Myhrvold, and the USNO funded the array-technology development and initial construction phase to implement the ATA-42. Completion of the ATA-350 requires additional private and federal partners. Absent a funding projection timeline, it is difficult to estimate the actual cost of the ATA-350, but our best estimate is that it will be somewhere between $1 / 6$ and $1 / 5$ the cost of building a traditional antenna of this size for $\mathrm{cm} \lambda$ astronomy. This is encouraging as we look to the future of SETI and centimeter-decimeter radio astronomy and to the Square Kilometre Array, a project to build an international observatory with 100 times the collecting area of the ATA. More work is needed to bring down the unit cost of $A_{\text {eff }} / T_{\text {sys }}$ but greater opportunities of scale, the direct involvement of industrial partners, and the lessons learned from actually building a Large N - Small D array of small telescopes like the ATA are suggesting several potentially successful pathways 
forward. We need to get through today and finish the ATA-350, before we can enjoy the promises of tomorrow. So at this General Assembly, I am hoping that 42 may soon turn out to be the answer to life-the-Universe-and-SETI! But just in case it doesn't, I am reduced to holding out my hands and quoting Jodie Foster - we know what to do, now all we need is the money.

Moore's Law improvements have helped OSETI programs every bit as much as they have enabled the ATA. At Harvard, Paul Horowitz and his students are just beginning to collect data with a new, dedicated, OSETI telescope for a survey of the $60 \%$ of the northern sky visible from the Oak Ridge Observatory in Massachusetts (Horowitz 2006). The telescope is housed in a building enthusiastically constructed with student labor, featuring a roll-back roof and a removable section in the south-facing wall that accommodates drift scans with only a single axis of rotation. The 72-inch primary and 36-inch secondary mirrors have been manufactured inexpensively by fusing glass over a spherical form and then polishing, because the system does not require image quality optics. The detection system is based on eight pairs of 64-pixel Hamamatsu fast photo-diodes, and custom electronics to permit real-time pixel by pixel intercomparison for coincidence detection to eliminate false positives. This new telescope will search for powerful transmitters from a large collection of stars by conducting meridian transit scans of the sky in $1 .{ }^{\circ} 6 \times$ $0^{\circ} .2$ strips (with a dwell time, due to the Earth's rotation, of about one minute). The sky visible from that site can be scanned in approximately 150 clear nights. The survey sensitivity should be adequate to detect laser pulses from the analog of a Helios-class laser being transmitted through a $10 \mathrm{~m}$ telescope up to a distance of 1000 ly. To improve the sensitivity of OSETI searches, larger aperture antennas are needed. To make these searches affordable, it is important to consider commensal observations with $10 \mathrm{~m}$ class optical light buckets being built for other purposes. Gamma-ray, Cherenkov-detecting telescopes are possibilities. SETI signals can be distinguished from the extended shower events in single dishes, such as the Whipple $10 \mathrm{~m}$ telescope, by means of the individual image shapes (Holder et al. 2005), or in the case of array like VERITAS, if a fast trigger could be developed, then single pixel events originating at the same point in the sky could be recovered as candidate SETI signals rather than being discarded as noise. Opportunities for commensal OSETI observations should also be considered for future terrestrial planet imaging missions as well as the overwhelmingly-large class of new groundbased telescopes being proposed for the future.

\section{Broadcasting}

This presentation has considered only one side of the communication equation - that of the receiver. However, if everybody listens and nobody transmits, then communication will not occur. Referring back to our asymmetric position vis á vis any other technological civilization in the Milky Way - we are the youngest - it is appropriate that as we listen first. Transmission is a harder job than receiving. It is more expensive to build and power transmitters, and it is also more expensive in the cultural currencies of diplomacy, ethics, and politics. Today we lack any form of global governance, we have no mechanism with which to reach concensus on whether we should transmit (either $a b$ initio or in response to a detected signal) and what we should say, and who should say it on behalf of the human species on planet Earth. And we are impatient. While a listening strategy could yield results immediately. If transmission is required to elicit a reply, then the times involved are, at a minimum, the roundtrip light travel times between us and the intended target. As a species, we are also not very good at long term commitments. We focus on 2,5 , and 10 year plans, are beginning to come to grips with the environmental consequences 
hundreds of years in the future of actions we take today, but we are ill-equipped to commence and continue a transmission strategy whose duration needs to be measured in Galaxy-crossing times at a minimum, and potentially much longer if we wish to include technologies that may emerge after us. Listening comes first - any other technologies will have done that when they were young. Now that they are older, and have successfully grappled with the questions above, they may have assumed the burden of transmission, and so should we when we become older.

\section{Finding our replacements}

Kids really do like dinosaurs, ghosts, creepy-crawly things, and ET. This gives us a wonderful hook on which to hang a science education. Astrobiology is a particularly appealing story to tell in the classroom. It allows students to put themselves into the big picture and encourages them to reach across discipline boundaries to embrace whatever body of knowledge and tools that can help solve a problem. At the SETI Institute, where my colleagues and I have embarked on an exploration of the cosmic haystack that could well take far more than our lives to systematically conclude, we think a lot about how to keep the searching going after we retire or pass away. We think about it in financial terms - how do we establish an endowment that will be viable into the indefinite future. And we think about it in human terms - where will we find the next generation of SETI researchers. Though it is now far closer to being a mainstream concept than when I first got hooked on SETI, it is still distinct enough that we cannot assume that this exploration will endure through the traditional route of generation after generation of university students being educated and then educating more students within the larger academic structure. So we try to improve the odds a bit by developing formal curriculum materials for young students, to try to counter Herman Bondi's observation when told that all young children were fearlessly curious - we have a cure for that, it's called education! Our latest attempt is a year-long integrated science curriculum for ninth graders, called Voyages Through Time, and it has a subtitle Everything Evolves. So we're not standing on the sidelines, but trying to get involved where it counts to promote future science literacy for the next generation.

SETI might succeed in my lifetime, or in my grandaughter's, or never. There is no satisfactory way to make an estimate. The wisest summary still remains the last sentence in the original 1959 Nature journal article: The probability of success is difficult to estimate, but if we never search the chance of success is zero.

So I invite you to stay tuned, because some of us are determined to keep searching!

\section{References}

Backus, P.R. 2006, in preparation

Beatty, J. T., Overmann, J., Lince, M. T., Manske, A. K., Lang, A. S., Blankenship, R. E., Van Dover, C. L., Martinson, T. A., \& Plumley, F. G. 2005, Proc. Nat. Acad. Sci., 102, 9306

Beaulieu, J.-P., Bennett, D. P., Fouque, P., Williams, A., \& Dominik, M. 2005, Nature, 439, 437 Bell Burnell, J. S. 1977, Annals NY Acad. Sci., 302, 685

Breezee, J., Cady, N., \& Staley, J.T. 2004, Microb Ecol., 300, 4

Cabrol, N. A., Grin, E. A., Prufert-Bebout, L., Rothschild, L, \& Hock, A. N. 2005, Astrobiology, 5,305

Cobb, J., Lebofsky, M., Werthimer, D., Bowyer, S., \& Lampton, M. 2000, in: G.A. Lemarchand \& K.J. Meech (eds.), Bioastronomy '99: A New Era in Bioastronomy, ASP-CS, 213, 485

Cocconi, G., \& Morrison, P. 1959, Nature, 184, 844 
Cohen, R. J., Downs, G., Emmerson, R., Grimm, M., Gulkis, S., Stevens, G., \& Tarter, J. C. 1987, MNRAS, 225, 491

Dalton, J. B., Mogul, R., Kagawa, H. K., Chan, S. L., \& Jamieson, C. S. 2003, Astrobiology, 3, 505

Darling, D. 2001, Life Everywhere: The Maverick Science of Astrobiology (New York: Basic Books)

DeBoer, D., Welch, W. J., Dreher, J. D., Tarter, J. C., Blitz, L. Davis, M. D., Fleming, M., Bock, D., Bower, G., Lugten, J., Girmay-Keleta, G., D'Addario, L., Harp, G., Ackermann, R., Weinreb, S., Engargiola, G., Thornton, D., \& Wadefalk, N. 2004, Proc. SPIE, 5489, 1021

De Duve, C. 1996, Vital Dust: Life As a Cosmic Imperative (New York: Basic Books)

Dick, S. J. 1984, Plurality of Worlds: The Extraterrestrial Life Debate from Democritus to Kant (Cambridge: CUP)

Drake, F. D. 1960, Sky \& Telescope, 39, 140

Fisher, C. R., MacDonald, I. R., Sassen, R., Young, C. M., Macko, S., Hourdez, S., Carney, R., Joy, S., \& McMullin, E. 2000, Naturwissenschaften, 87, 184

Gold, T. 1998, The Deep Hot Biosphere (Berlin: Springer)

Gott, J. R. 1993, Nature, 363, 315

Greene, B. 2000, The Elegant Universe: Superstrings, Hidden Dimensions, and the Quest for the Ultimate Theory (Vintage Press)

Harwit, M. 1981, Cosmic Discovery: The Search, Scope, and Heritage of Astronomy (New York: Basic Books)

Harwit, M. 2003, ApJ, 597, 1266

Holder, J., Ashworth, P., LeBohec, S., Rose, H.J., \& Weekes, T. C. 2005, Proc. 29th Int. Cosmic Ray Conf., 5, 387

Horowitz, P., Coldwell, C., Howard, A., Latham, D., Stefanik, B., Wolff, J., \& Zajac, J. 2006, preprint <http://seti.harvard.edu/oseti/oseti.pdf>)

Howard, A. B., \& Horowitz, P. 2001, Proc. SPIE, 4273, 153

Howard, A. W., Horowitz, P., Wilkinson, D. P., Coldwell, C. M., Groth, E. J., Jarosik, N., Latham, D. W., Stefanik, R. P., Willman, A. J. Jr., Wolff, J., \& Zajac, J. M. 2004, ApJ, 613,1270

Kashefi, K., \& Lovley, D. R. 2003, Science, 301, 394

Kurzweil, R. 2005, The Singularity Is Near: When Humans Transcend Biology (Viking Press)

Leger, A., Ollivier, M., Altwegg, K., \& Woolf, N. J. 1999, A\&A, 341, 304

Lemarchand, G. A. 1994, Ap. Space Sci., 214, 209

Levin, G. V. 1997, SPIE Proc., 3111, 146

Lineweaver, C., Fenner, Y., \& Gibson, B. 2004, Science, 303, 59

Lovelock, J. 1965, Nature, 207, 568

Mallove, E. F. MIT Tech Talk, Nov. 7

Mattimore, V., \& Battista, J. R. 1996, J. Bacteriology, 178, 633

McKay, D. S., Gibson, E. K. Jr., Thomas-Keprta, K. L., Vali, H., Romanek, C. S., Clemett, S. J., Chillier, S. E. F., Maechling, C. R., \& Zare, R. N. 1996, Science, 273, 924

Montanes-Rodriguez, P., Palle, E., Goode, P. R., Hickey, J., \& Koonin, S. E. 2005, ApJ, 629, 1175

Mumma, M. J., Novak, R. E., Hewagama, T., Villanueva, G. L., Bonev, B. P., DiSanti, M. A., Smith, M. D., \& Dello Russo, N. BAAS, 37, 669

Pelli, D. G., \& Chamerlain, S. C. 1989, Nature, 337, 460

Raymond, S. N., Mandell, A. M., \& Sigurdsson, S. 2006, Science, 313, 1413

Roadcap, G. S., Sanford, R. A., Qusheng, J., Pardinas, J. R., \& Bethke, C. M. 2006, Ground Water, 44, 511

Rothschild, L. J., \& Mancinelli, R. L. 2001, Nature, 409, 1092

Rothschild, L. J., Giver, L. J., White, M. R., \& Mancinelli, R. L. 1994, J. Phycol., 30, 431

Schulze-Makuch, D., Grinspoon, D. H., Abbas, O., Irwin, L. N., \& Bullock, M. A. 2004, Astrobiology, 4, 11

Segura, A., Kasting, J. F., Meadows, V., Cohen, M., Scalo, J., Crisp, D., Butler, R. A. H., \& Tinetti, G. 2005, Astrobiology, 5, 706 
Shelley, M. 1818, Frankenstein, or, The Modern Prometheus (London: Lackington, Hughes, Harding, Mavor \& Jones)

Squyres, S. W., Knoll, A. H., Arvidson, R. E., Clark, B. C., \& Grotzinger, J. P. 2006, Science, 313, 1403

Tarter, J. C. 2001, ARAA, 39, 511

Tinetti, G., Meadows, V. S., Crisp, D., Fong, W., Fishbein, E., Turnbull, M., \& Bibring, J.-P. 2006, Astrobiology, 6, 34

Townes, C. H., \& Schwartz, R. N. 1961, Nature, 192, 348

Turnbull, M. C., \& Tarter, J. C. 2003, ApJS, 145, 181

Vidal-Madjar, A., Desert, J.-M., Levavelier des Etangs, A., Hebrard, G., Ballester, G. E., Ehrenreich, D., Ferlet, R., McConnell, J. C., Mayor, M., \& Parkinson, D. C. 2004, ApJ (Letters), 604, L69

Ward, P. \& Brownlee, D. 2001, Why Complex Life is Uncommon in the Univere (Berlin: Springer)

Woolf, N. J., Smith, P. S., Traub, A., \& Jucks, K. W. 2002, ApJ, 574, 430 